\title{
Modelación de la distribución de especies y ecosistemas en el tiempo y en el espacio: una revisión de las nuevas herramientas y enfoques disponibles ${ }^{1}$
}

\author{
Patricio Pliscoff ${ }^{2}$ y Taryn Fuentes-Castillo ${ }^{3}$
}

\begin{abstract}
RESUMEN
Este artículo tiene como objetivo presentar el marco conceptual y metodológico en que se desarrollan las técnicas de modelos de distribución para especies y ecosistemas. Se hace una revisión histórica de los conceptos que fundamentan estas técnicas y se presentan las principales etapas metodológicas involucradas en estos análisis. Se discute cómo estos enfoques son de gran utilidad para el desarrollo de nuevas preguntas en el campo de la biogeografía y de la conservación biológica. Finalmente, se presenta una aplicación de técnicas de modelos de distribución, tomando como caso de estudio Beilschmiedia miersii (belloto del norte). Esta revisión conceptual y metodológica, así como el ejemplo aplicado, buscan clarificar la utilidad y el potencial que tienen los modelos de distribución, con el objetivo de invitar a la investigación en biogeografía y así seguir avanzando en el conocimiento de los patrones espaciales y temporales de la distribución de los organismos.
\end{abstract}

Palabras clave: Modelos de distribución de especies/ecosistemas, nicho ecológico, biogeografía de la conservación, cambio climático.

\begin{abstract}
This article aims to present the conceptual and methodological framework in which models techniques for species and ecosystems distribution are developed. An historical review of concepts behind these techniques is made as well as the presentation of the major methodological steps involved in these tests. A discussion on how these approaches are useful for the development of new questions in the field of biogeography and biological conservation is generated. Finally, an application of distribution modeling techniques, using the specie Beilschmiedia miersii (belloto Del Norte) as a study case, is presented. This conceptual and methodological review as well as the example applied, seeks to clarify the usefulness and potential of distribution models techniques, with the objective to go forward in biogeography research and thus, farther progress in understanding spatial and temporal patterns of organism's distribution.
\end{abstract}

Key words: Distribution models species/ecosystems, ecological niche, conservation biogeography, climate change.

1 Artículo recibido el 27 de diciembre de 2010, aceptado el 15 de marzo de 2011 y corregido el 23 de marzo de 2011.

2 Laboratorio de Ecología Espacial, Universidad de Lausanne (Suiza). E-mail: patricio.pliscoff@unil.ch

\footnotetext{
3 Facultad de Ciencias Forestales y de Conservación de la Naturaleza, Universidad de Chile (Chile). E-mail: tarfuentes@uchile.cl
} 
Identificar los patrones espaciales y temporales de la distribución de los seres vivos sobre el planeta ha sido desde sus inicios el centro del estudio de la biogeografía. Es así como la identificación y caracterización de "áreas de distribución" (Axelius, 1991; Morrone, 2009) se ha señalado como el hilo conductor en el desarrollo del conocimiento biogeográfico (Lomolino et al., 2010). Desde el viaje de Humboldt y Bonpland hacia América, pasando por la "Geografía botánica Rasonée" de Agustín de Candolle en 1885, se inició una tradición investigativa que ha estudiado la relación entre la distribución de las especies vegetales y el clima.

La geobotánica en los inicios del siglo XX sistematizó el conocimiento de la distribución de las especies vegetales y su relación con el clima, al complementar información obtenida en terreno con el nuevo conocimiento cartográfico del globo terrestre. Estos avances permitieron construir una relación causal entre la distribución geográfica de las especies vegetales y la distribución espacial del clima. Posteriormente, desde la ecología, surge el concepto de nicho ecológico, ampliando fuera del ámbito de la botánica la relación entre los seres vivos y los factores abióticos. Este concepto tiene su origen en los trabajos de los ecólogos Johnson, Grinnell y Elton, donde se buscaba establecer el rol de las especies e identificar la subdivisión del ambiente (hábitat) que era ocupado por una especie. La concepción de nicho más utilizada es la desarrollada por Hutchinson (1957), donde se refiere al nicho como un hipervolumen de $\mathrm{n}$-dimensiones donde se encuentran las condiciones ambientales (factores) en las cuales la especie puede sobrevivir. Esta definición es además clave en la conceptualización de los modelos de distribución de especies que se detallarán más adelante, ya que permite relacionar directamente el espacio geográfico a través de la noción de espacio multidimensional de $n$ variables, donde una especie se encuentra habitando o potencialmente lo podría hacer. Es aquí donde se encuentra la distinción entre nicho potencial o fundamental y nicho realizado, donde el primero indica al espacio total de las variables y el segundo especifica el espacio geográfico donde efectivamente habita la especie.

\section{Del concepto de nicho ecológico a los modelos de distribución}

El concepto de nicho se abordó en la ecología, tanto en su concepción matemática (MacArthur, 1972) como experimental (Austin et al., 1990). A finales de la década de los 70 se comenzaron a desarrollar los primeros modelos teóricos en los cuales se buscaba definir el nicho en términos de su distribución espacial (Kessell, 1979). Durante la década de los 80 comienzan a generarse las primeras aproximaciones con carácter predictivo a distintos niveles de organización (especies, comunidades). Box (1981) es el primero en establecer un modelo global para la predicción de la distribución de los biomas. En Australia comienzan los trabajos de Austin (1987), aplicando modelos estadísticos para cuantificar la relación vegetación-ambiente (gradientes), y Nix (1986) establece los fundamentos para el algoritmo BIOCLIM, un algoritmo simple que se basa en operadores booleanos, que hasta el día de hoy es utilizado para generar modelos de distribución espacial de especies (Beaumont et al., 2005). Con el inicio de la masividad de los Sistemas de Información Geográfica en los años 90 y la mayor disponibilidad y manejo de grandes bases de datos, se dispone de nuevas herramientas para proyectar en el espacio ecológico y geográfico los modelos estadísticos de nicho. Es así como comienzan a surgir distintas aproximaciones estadísticas y programas computacionales que permiten determinar la distribución espacial de las especies y ecosistemas, basándose en datos de presencia/ausencia (Guisan y Zimmermann, 2000; Guisan y Thuiller, 2005; Elith y Leathwick, 2009).

En la actualidad, con las nuevas técnicas y programas computacionales disponibles, se pueden obtener en forma muy simple modelos de distribución, esto ha llevado a críticas (Jiménez-Valverde et al., 2008) que se preguntan si los modelos de distribución están efectivamente respondiendo a las preguntas ecológicas básicas sobre las cuales fueron definidos (Austin, 2007). Se señala que la existencia de técnicas estadísticas cada vez más complejas y el manejo de gran cantidad de información espacial, está desviando la pregunta central que buscan responder estas aproximaciones: cuáles son los factores que 
determinan la distribución de los seres vivos (McGill, 2010).

Los modelos de distribución de especies/ ecosistemas se han transformado en una de las áreas de investigación con mayor desarrollo en el campo de la biogeografía de la conservación (Richardson y Whittaker, 2010). Entendiendo este campo de estudios como la aplicación del conjunto de principios, teorías y análisis de la biogeografía a problemas concernientes a la conservación de la biodiversidad (Whittaker et al., 2005). La capacidad predictiva de los modelos de distribución de especies/ecosistemas tanto para proyectar el espacio geográfico-ecológico en el pasado y en el futuro, ha generado nuevas técnicas y herramientas, generando así un nuevo dinamismo a la disciplina biogeográfica (Guisan \& Thuiller, 2005).

\section{Técnicas de modelación}

Se pueden distinguir cuatro grandes familias de técnicas ${ }^{4}$ de modelación de la distribución geográfica de las especies/ ecosistemas: las relacionadas a modelos estadísticos de regresiones (Modelos Lineales Generalizados (GLM), Modelos Aditivos Generalizados (GAM)), los métodos de clasificación (Random Forest (RF), Boosted regresion trees (BRT)), los métodos de "sobre" (BIOCLIM, ENFA) y aquellos basados en algoritmos específicos (GARP, MAXENT). Además, se puede incluir como un nuevo enfoque los

\footnotetext{
4 Una revisión completa de los métodos disponibles se encuentra en Guisan \& Thullier (2005) y en Franklin (2009).
}

Cuadro $\mathrm{N}^{\circ} 1$

Comparación de algunas de las principales técnicas para la generación de modelos de distribución

\begin{tabular}{|c|c|c|c|c|c|c|c|c|}
\hline \multirow{2}{*}{$\begin{array}{c}\text { Técnicas } \\
\text { (BM = presente } \\
\text { en software } \\
\text { BIOMOD) }\end{array}$} & \multirow[b]{2}{*}{ Nombre completo } & \multirow[b]{2}{*}{ Referencia } & \multicolumn{6}{|c|}{ Estudios comparativos } \\
\hline & & & A & B & $\mathrm{C}$ & $\mathrm{D}$ & $\mathrm{E}$ & $\mathrm{F}$ \\
\hline ANN (BM) & Artificial Neural Networks & Lek et al. (1996) & & & & & + & \\
\hline $\begin{array}{l}\text { BIOCLIM } \\
(B M=S R E)\end{array}$ & Bioclimatic Envelope Algorithm & Busby (1991) & - & - & & & & \pm \\
\hline BRT (BM) & Boosting Regression Trees & Friedman (2001) & + & & & + & & \\
\hline CART (BM) & Classification And Regression Trees & Vayssieres et al. (2000) & & & - & - & \pm & \\
\hline ENFA & Ecological Niche Factor Analysis & Hirzel et al. (2002) & & & & & - & - \\
\hline GAM (BM) & Generalized Additive Models & Hastie \& Tibshirani (1990) & \pm & + & + & & \pm & \\
\hline GARP & Genetic Algorithm for Rule-set Production & Stockwell \& Peters (1999) & \pm & & - & & & + \\
\hline GDM & Generalised Dissimilarity Modelling & Ferrier et al. (2007) & + & & & & & \\
\hline GLM (BM) & Generalized Linear Models & McCullagh \& Nelder (1989) & \pm & & + & & \pm & \\
\hline MARS (BM) & Multivariate Adaptive Regression Splines & Friedman (1991) & \pm & & & - & & \\
\hline MAXENT & Maximum Entropy & Phillips et al. (2006) & + & + & & & & \\
\hline $\mathrm{RF}(\mathrm{BM})$ & Random Forest & Breiman (2001) & & & & + & & \\
\hline
\end{tabular}

Referencias para los estudios comparativos: $\mathrm{A}=$ Elith et al. (2006), B = Hijmans \& Graham (2006), C = Meynard \& Quinn (2007), D = Prasad et al. (2006), E = Segurado \& Araujo (2004), F = Tsoar et al. (2007). La evaluación de las técnicas es indicada como: -- (muy mal), - (mal), \pm (promedio) o + (buena).

Fuente: Modificado de Van Strien, 2008. 
programas que utilizan ensambles de técnicas para obtener modelos de consenso, buscando disminuir los sesgos y limitaciones propias del uso en forma individual de las técnicas estadísticas mencionadas. En el Cuadro $\mathrm{N}^{\circ}$ 1 se resumen las principales técnicas, los programas que las aplican y su rendimiento en las principales evaluaciones que se han realizado.

\section{Modelos estadísticos de regresiones}

Los métodos basados en regresiones múltiples han sido los más utilizados para modelar (Guisan et al., 2002), ya que permiten establecer de forma clara el modelo estadístico que describe la relación entre las variables descriptivas y la presencia conocida de las especies/ecosistemas. Además, en las evaluaciones comparativas entre técnicas de modelación, estos métodos han sido identificados como una de las técnicas de mejor resultado en la proyección sobre el espacio geográfico del modelo final (Elith et al., 2006).

\section{Métodos de clasificación}

Estos métodos se basan en clasificaciones basadas en reglas, donde se asigna una clase para cada combinación de las variables predictivas que generan el modelo de distribución (Guisan \& Zimmermann, 2000), algunas de las técnicas que se encuentran dentro de esta categoría, por ejemplo Random Forest (Breiman, 2001), han sido identificadas como las con mejores resultados al momento de discriminar la importancia de las variables para la definición del modelo final (Prasad et al. 2006), pero a su vez, la caracterización del conjunto de reglas que define el modelo final es muy compleja, por lo que se hace difícil de interpretar.

\section{Métodos de "sobre"}

Los métodos de "sobre" se basan en el establecimiento de combinaciones de rangos de valores mínimos y máximos, entre las variables utilizadas para la definición del nicho. Donde se define un espacio o "sobre" en que se da la combinación de valores deseada, dentro del espacio n-dimensional de las variables (Busby, 1991). Este es el enfoque más antiguo de modelación, pero se ha ido abandonando con el tiempo, ya que ofrece una definición muy poco flexible del espacio ambiental, sin tomar en cuenta los distintos tipos de interacciones que se dan entre las variables (por ejemplo colinealidad) o el efecto de la distancia geográfica entre las presencias (autocorrelación espacial).

\section{Algoritmos específicos para generar modelos de distribución}

En los últimos años ha tomado fuerza el uso de técnicas basadas en algoritmos específicos (máxima entropía, algoritmos genéticos). Han significado un aumento exponencial en el número de publicaciones y estudios asociados a la modelación de la distribución geográfica de las especies y ecosistemas, por la facilidad de su aplicación y la rapidez con que se pueden obtener resultados. En este contexto destaca el software MAXENT (Phillips et al., 2006; Elith et al., 2011) que, usando un algoritmo de máxima entropía, logra resultados robustos en términos de la proyección espacial de la distribución, especialmente cuando se cuenta con pocos datos de presencias, sin embargo, la relevancia de las variables ambientales y su evaluación estadística se mantiene en discusión (Peterson et al., 2007). Pese a ello su uso se ha masificado en los últimos años, siendo utilizado en prácticamente todas las aplicaciones de modelos de distribución (Baldwin, 2009). Existen también algunos casos en que MAXENT ha sido utilizado desde un enfoque de modelación de ecosistemas (Thuiller et al., 2005; Deblauwe et al., 2008; Riordan \& Rundel, 2009).

\section{Ensambles de técnicas}

Una reciente aproximación es utilizar "ensambles de técnicas" (Araujo \& New, 2007; Marmion et al., 2009), con el objetivo de disminuir las incertidumbres asociadas a los modelos de distribución, especialmente en relación a la validez estadística de los resultados. BIOMOD (Thuiller, 2009) es un ejemplo de ensamble de técnicas, que permite aplicar a la vez nueve técnicas de modelación. Es un software programado en el lenguaje estadístico $R$ que ha sido utilizado principalmente para analizar proyecciones de la distribución actual bajo escenarios de cambio climático, análisis del nivel de protección actual y futuro de especies con problemas de conservación (Le Maître et al., 2008; Barbet- 
Massin et al., 2009; Parviainen et al., 2009; Marini et al., 2010).

\section{Cómo modelar}

Los modelos de distribución se generan usando dos tipos de fuentes de información: los datos de presencia o ausencia de las especies/ecosistemas que se quiere modelar y las variables descriptoras que definirán el espacio ambiental, donde se distribuirá en el espacio geográfico. La técnica de modelación seleccionada establecerá una relación entre la posición geográfica de la información de presencia o ausencia y el rango de valores del conjunto de variables donde se ubican estos puntos. Esta relación es usualmente llamada el ajuste del modelo de la ecuación, regla de clasificación o algoritmo seleccionado como técnica (Figura $N^{0} 1$ ).

\section{Datos de presencias (ausencias)}

La forma más básica de obtener los datos de presencia, es generando la información en terreno mediante GPS u otros tipos de herramienta de adquisición de datos, o recurriendo a las fuentes directas de información de especies, como son los museos o herbarios. También se puede recopilar información de forma indirecta mediante el uso de bases de datos de libre acceso, que cada vez adquieren mayor importancia. Un ejemplo es la base de datos de especies GBIF (Global Biodiversity Information Facility ${ }^{5}$ ) que recopila la información de colectas para todo tipo de organismos, desde diferentes fuentes (museos,

\footnotetext{
5 Se puede acceder a ella a través del sitio web http://
} www.gbif.org

Figura $\mathrm{N}^{\mathrm{o}} 1$

Pasos metodológicos para la generación de modelos de distribución de especies/ecosistemas

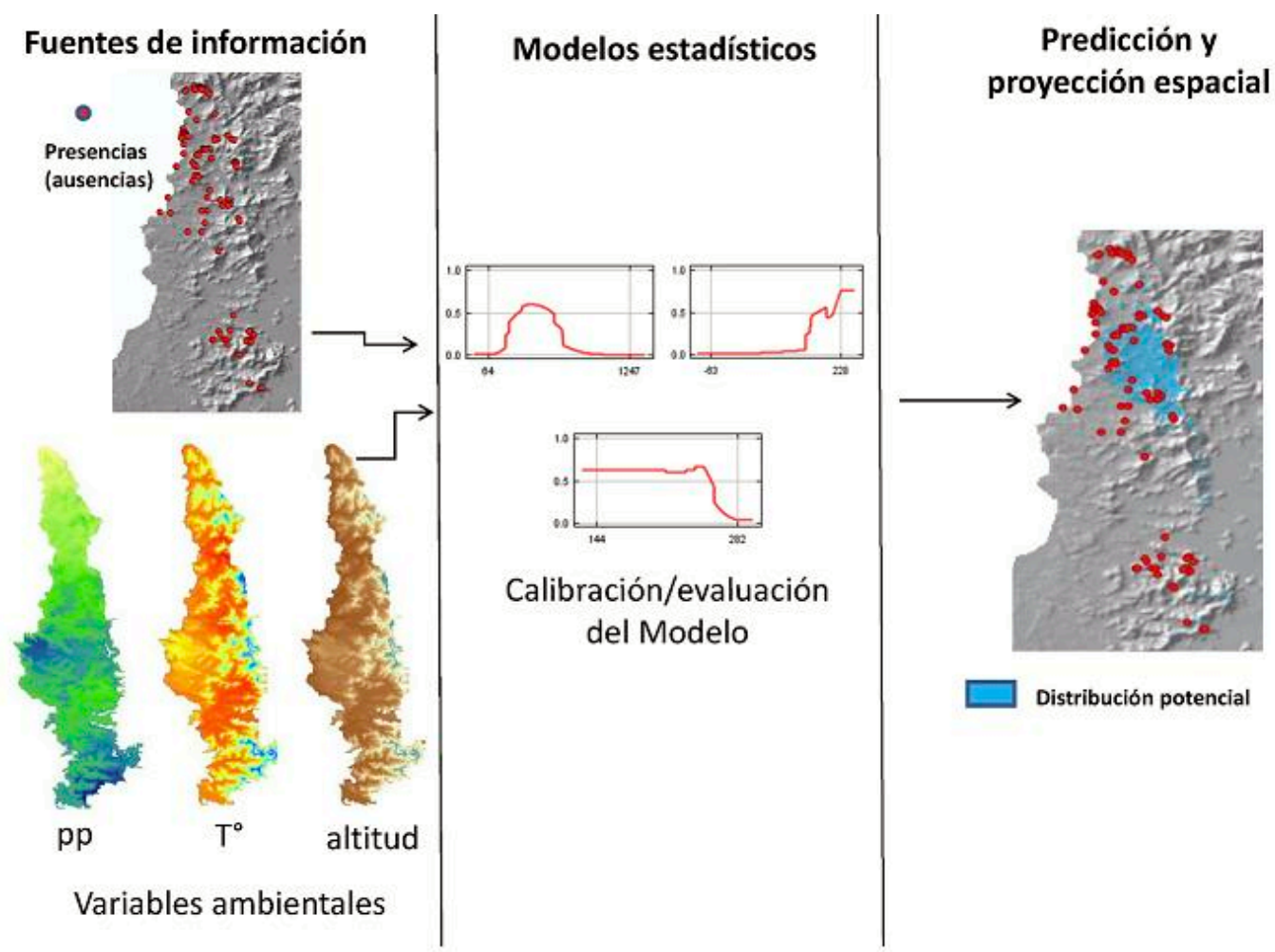

Fuente: Modificado de Guisan et al., 2002. 
herbarios, estudios, entre otros). El uso de estas fuentes de información estandarizadas, aunque son de gran utilidad por su fácil acceso y uso, son criticadas debido a que no toda la información posee criterios mínimos de revisión, lo que genera tanto errores de tipo taxonómico (correcta identificación) como también geográficos (errores en la localización) (Newbold, 2010). Este aspecto produce la primera fuente de error en los modelos, generando errores independientes de las técnicas utilizadas (Feeley \& Silman, 2009).

Cuando existe la posibilidad de levantar información de la presencia del organismo que se quiere modelar, es necesario fijar una correcta estrategia de muestreo. La definición de cómo levantar los datos tiene un efecto directo en el desempeño final del modelo (Edwards et al., 2006) y a su vez puede ser de gran relevancia si lo que se quiere es abordar preguntas específicas como la identificación de especies raras (Le Lay et al., 2010). En general, independiente de la escala de trabajo definida se deben seguir criterios de heterogeneidad en la selección de sitios de muestreos (privilegiar el muestreo en distintos ambientes) y de homogeneidad en la colecta (definir distancias regulares de muestreo con el fin de evitar la posterior correlación espacial de las colectas). La posibilidad de obtener ausencias (identificar espacialmente los sitios donde la entidad natural analizada no se encuentra presente) puede mejorar sustancialmente el resultado final de los modelos (Guisan \& Zimmermann, 2000).

Existen técnicas de modelación que incorporan las ausencias, lo que permite una mejor discriminación de las variables descriptoras y por lo tanto un mejor ajuste final del modelo. Aunque lo más común es trabajar solo con presencias, debido a que existen programas con técnicas especialmente adaptadas para la utilización de pocos registros de presencia. En relación al número ideal de presencias por unidad natural analizada, varía de acuerdo al objetivo planteado, de igual manera de las técnicas que se quiere utilizar. Criterios de números mínimos para obtener modelos estadísticamente robustos se han planteado en la literatura, por ejemplo, se ha establecido que sobre 30 (Wisz et al., 2008) o 50 (Kadmon et al., 2003) registros por especie se pueden obtener modelos estadísticamente válidos. No obstante, el criterio a seguir es incorporar el mayor número de presencias disponibles, independiente de la calidad de la información, ya que es posible evaluar los modelos según la calidad de los datos de presencia incorporados con posterioridad.

\section{Variables ambientales}

El siguiente paso es la selección de las variables ambientales antes de la aplicación de las técnicas de modelación. El elegir un número de variables adecuado y que releven la información más importante de la entidad natural analizada, es quizás el paso más crítico en el proceso de los modelos de distribución. El resultado final está completamente determinado por esta elección. Las variables que se deben utilizar dependen del tipo de especie que se esté analizando y de los objetivos que busca la identificación y caracterización del espacio ambiental donde se distribuye.

Existen principalmente dos tipos de variables que se utilizan comúnmente, las variables climáticas (bioclimáticas) y las variables topo-edáficas. Las primeras son las más utilizadas, independiente del tipo de entidad natural, principalmente por la facilidad de su obtención, ya que existen bases de datos globales a alta resolución $(\sim 1 \mathrm{~km})$ de libre acceso y uso. Una de las estrategias de selección más utilizada es trabajar con un set de variables bioclimáticas predefinidas, como es el caso de las 19 variables bioclimáticas disponibles en la base de datos global de superficies climáticas Worldclim (Hijmans et al., 2005).

Sobre el uso de este tipo de variables $y$ el efecto que pueden tener sobre los resultados finales, existen varias revisiones $y$ evaluaciones (Elith et al., 2002; Van Niel et al., 2004; Dormann et al., 2008). Es posible señalar, para el caso de Chile, que estas bases de datos climáticos globales (por ejemplo Worldclim) se deben utilizar criteriosamente ya que en evaluaciones realizadas se señala que poseen problemas en los valores de las variables climáticas, especialmente en aquellas zonas del país donde la cobertura de estaciones meteorológicas es menor (zona norte del país). Respecto a otros tipos de variables como las topo-edáficas o derivadas de imágenes satelitales, como las coberturas 
de uso de suelo o índices de productividad, se ha identificado que pueden ser de gran utilidad para obtener modelos con un mayor ajuste (Zimmermann et al., 2007; Randin et al., 2009a). Especialmente en el caso de las topo-edáficas, se ha probado que su uso en forma conjunta con los datos climáticos, puede mejorar el resultado de los modelos a escalas espaciales de mayor detalle (Randin et al., 2009b).

\section{Pasos para generar modelos de distribución}

Una vez definida la información de las presencias y variables, se debe seleccionar la técnica de modelación más adecuada para las preguntas que se quieran responder y proceder a los pasos básicos que involucra el trabajo con modelos: calibración, evaluación, predicción y proyección espacial (Figura No 1 ).

\section{Calibración del modelo}

La calibración del modelo se refiere a los análisis estadísticos asociados a buscar el ajuste del modelo que posteriormente se proyectará en el espacio geográfico. El tipo de calibración dependerá de la técnica que se esté utilizando. El desarrollo de la aplicación de las técnicas en los últimos años ha permitido realizar evaluaciones con diferentes tipos de organismos, a distintas escalas espaciales y sobre diferentes espacios geográficos (Manel et al., 2001; Hernández et al., 2006; Hirzel et al., 2006; Elith et al., 2006; Elith y Graham, 2009). Estas evaluaciones sirven como guía para la selección de la técnica con la que posteriormente se calibrarán los modelos. Existen, además, algunas herramientas muy útiles para analizar los resultados de los modelos, una de ellas son las superficies de respuestas, que son gráficos que muestran cómo se distribuyen las presencias sobre los valores de las variables descriptoras seleccionadas. Finalmente, la posibilidad de uso de softwares que calibran automáticamente los modelos para un conjunto de distintas técnicas, como BIOMOD (Thuiller, 2009), ayuda también de gran forma para el análisis de los resultados de las calibraciones de los modelos, ya que permite interpretar estos resultados con distintas réplicas que corres- ponderían a cada técnica, lo que favorece la interpretación de los resultados.

\section{Evaluación del modelo}

La evaluación o validación se enfoca en la caracterización de la confiabilidad de los resultados de los modelos, más que en la definición de uno como correcto o incorrecto (Allouche et al., 2006). La forma correcta de evaluar el resultado sería comparándolo con un conjunto de presencias independientes al que se está usando, lo que es difícil de realizar porque se tienen datos de presencia limitados, esto aborda procediendo a dividir el conjunto de datos en dos set: uno de calibración y otro de evaluación (Guisan \& Zimmermann, 2000). La forma más básica de evaluar un modelo consiste en generar una matriz de confusión o error, donde las ausencias y presencias predichas se comparan con las ausencias y presencias reales. Esto permite calcular indicadores para evaluar los modelos, como la sensitividad (porcentaje de positivos verdaderos correctamente predichos) y la sensibilidad (porcentaje de negativos verdaderos correctamente predichos) (Hirzel et al., 2006).

Hay, también, otras medidas de precisión de los modelos, donde un modelo es un mapa con valores continuos de probabilidad presencia, la forma más básica es reclasificar el mapa de valores continuos, basados en un valor de corte o umbral determinado, para luego analizar cómo los puntos conocidos de las presencias se distribuyen en este nuevo mapa reclasificado en forma binaria (Liu et al., 2005; Jiménez-Valverde \& Lobo, 2007; Freeman \& Moisen, 2008). Valores sobre el umbral determinado se estiman como presencia, y valores bajos como ausencia de la entidad en estudio.

Existen análisis estadísticos Ilamados umbral-independiente, esto quiere decir que se aplican para evaluar la precisión del modelo sobre el mapa de valores continuos, sin la necesidad de calcular un valor de corte o umbral (Manel et al., 2001). Los de mayor utilización son AUC, Kappa y TSS. El método umbral-independiente más conocido es el AUC (Area under the curve) (Dodd \& Pepe, 2003), que se obtiene comparando la proporción de falsos y verdaderos positivos, es decir, desplegando en dos ejes X e Y, la propor- 
ción de falsos positivos (1-especificidad) y en el eje Y la proporción de positivos verdaderos (sensitividad). La precisión de un modelo es mayor cuando la proporción de positivos verdaderos es mayor a la de positivos falsos, lo que se expresa gráficamente en una curva que mientras mayor sea su precisión, más se acercará a la esquina izquierda del gráfico, generando un "área de curva" mayor. Esto es lo que refleja el AUC; se estima que valores sobre 0,9 de AUC dan cuenta de modelos con alta precisión y valores entre 0,7 y 0,9 indican modelos con buena precisión (Thullier et al., 2009), pero se debe tener en cuenta que el valor del AUC depende del número de presencias y del tamaño total del área de estudio (Lobo et al., 2008).

\section{Predicción y proyección espacial}

El paso final en la generación de un modelo de distribución, es la proyección en el espacio geográfico del modelo que ha sido calibrado y evaluado. En este paso los valores continuos de probabilidad, o los valores binarios obtenidos de un umbral, son transferidos al espacio geográfico, por lo que se pueden representar en forma cartográfica. El modelo de distribución se puede proyectar en el espacio actual, utilizando variables descriptoras actuales, pero también se puede proyectar en el futuro o en el pasado, utilizando variables descriptoras que correspondan a los tiempos que se quieran analizar. Este proceso de transferencia temporal del modelo de distribución requiere de enfoques diferentes de calibración y evaluación. En la actualidad el enfoque más común de los trabajos de modelos de distribución involucra la proyección actual de la entidad natural analizada y su posterior proyección bajo un escenario de cambio climático. Para esto existen modelos climáticos globales, tanto para escenarios futuros y pasados (Hijmans et al., 2005), que sirven como fuentes para crear conjuntos de variables descriptoras bajo diferentes ventanas de tiempo. El proyectar distribuciones presenta nuevos desafíos para la biogeografía, donde se abren preguntas que antes no podían ser formuladas ni menos evaluadas, ya que no se contaba con las fuentes de datos necesarios; por ejemplo, la pregunta sobre si el nicho de una especie se mantiene en el tiempo y espacio, es un tema que está cobrando relevancia ya que involucra aspectos evolutivos (conser- vación del nicho) y ecológicos (estabilidad del nicho) (Wiens \& Graham, 2005; Pearman et al., 2008b).

\section{¿Qué pasará en el futuro? Proyectando los efectos del cambio climático}

Los modelos de distribución se han transformado en una herramienta esencial para la evaluación del efecto del cambio climático sobre las especies y ecosistemas (Dormann, 2007; Sinclair et al., 2010). El uso de este enfoque se está propagando entre expertos de distintos tipos de organismos, ya que en la actualidad es posible solo contando con un número menor de registros de colectas de especies, proyectar la distribución bajo distintos escenarios de cambio climático y en distintas ventanas de tiempo. Desde el punto de vista de la distribución actual es posible identificar incertidumbres asociadas a la generación de los modelos de distribución (fuentes de información de presencias, selección de variables adecuadas, diferencia entre técnicas, entre otros). $\mathrm{Al}$ proyectar en el futuro, se agregan nuevas incertidumbres relacionadas por ejemplo al comportamiento de las variables climáticas (que son las variables descriptivas utilizadas en las proyecciones), ya que se asume un cambio lineal en el comportamiento de una variable. La posible existencia, dada las variaciones climáticas proyectadas, de climas "no análogos", es decir, de combinaciones de variables que den cuenta de climas que no existen en la actualidad o que son nuevos para una determinada zona geográfica en particular (Williams et al. 2007; Williams \& Jackson, 2007), agrega otra dificultad a las proyecciones de cambio climático de las distribuciones de las especies/ ecosistemas. Nuevas versiones de programas de modelos de distribución permiten incorporar elementos dentro del análisis de las proyecciones futuras (Elith et al., 2010). Otra incertidumbre asociada a la proyección de las distribuciones bajo escenarios de cambio climático es la no inclusión del cambio de uso del suelo en los modelos (Franklin, 2009); es posible que el efecto de la desaparición y fragmentación de los hábitats naturales, producto del cambio de uso de suelo, sea temporalmente más relevante para las distribuciones actuales que la evolución del espa- 
cio climático. La mayoría de los trabajos que proyectan la distribución bajo escenarios de cambio climático, buscan resolver preguntas relacionadas con conservación biológica, evaluando el riesgo de extinción y la evolución del estado de conservación. Recientemente se han acoplado estos modelos con la información genética y poblacional de las especies (Kozak et al., 2008; Swenson, 2008) y también se están usando las proyecciones para evaluar los sistemas de reservas actuales y proponer nuevos diseños (Vos et al., 2008; Rose \& Burton, 2010).

\section{Aplicaciones en biogeografía histórica: ¿el espacio geográfico y el espacio ecológico evolucionan juntos?}

De la misma forma en que los modelos de distribución se pueden proyectar en el tiempo y el espacio futuros, es posible retroyectar la distribución en tiempos pasados (Yesson \& Culham, 2006; Pearman et al., 2008a; Willis et al., 2009; Graham et al., 2010). Para esto se deben evaluar dos conceptos diferentes, que al analizar la evolución y distribución de las especies cobran un valor fundamental para definir los nichos o espacios climáticos. Estos son el concepto de estabilidad y conservación de nicho (Nogués-Bravo, 2009). El primero se refiere a la posibilidad de que las especies/ecosistemas puedan mantener en el tiempo las condiciones climáticas en que persisten, y la conservación del nicho se relaciona con la posición de las especies dentro de su árbol filogenético, donde las especies que tienen más cercanía dentro del árbol deberían tener un espacio climático más similar. Si no se consideran estos dos conceptos, los resultados que se obtienen de la retroyección de las distribuciones en el pasado carecen de robustez.

Existen diferentes métodos que permiten evaluar la estabilidad y la conservación del nicho, para posteriormente poder definir las áreas más propicias para la distribución de una especie y su comportamiento en el tiempo y el espacio. El definir el comportamiento de la distribución en el pasado permite conocer antecedentes claves tanto para caracterizar de mejor forma la distribución actual como para hipotetizar cómo serán los cambios esperables en el futuro (Ficetola et al., 2010; Benito Garzón et al., 2008).

La existencia del registro fósil es de gran utilidad para la validación de los modelos pasados, sirviendo como muestras independientes en la evaluación de los modelos o también para identificar sesgos en las definiciones de las áreas de distribución pasadas, ya que pueden representar localidades erradas de la distribución que se quiere caracterizar en un tiempo determinado (Flantua et al., 2007).

Se han aplicado estos métodos para identificar refugios biogeográficos (Ashcroft, 2010), definir rutas de migración (MartínezMeyer et al., 2004), evaluar hipótesis de conservación de nicho (Martínez-Meyer et al., 2004; Peterson \& Nyari, 2008; Evans et al., 2009; Carnaval et al., 2009) y para la definición de áreas de distribución en tiempos pasados (Benito Garzón et al., 2007). El campo sigue en desarrollo así como el avance de nuevas técnicas que permitan mejorar o cuantificar la incertidumbre asociada a estos métodos (Smith \& Donoghue, 2010). Un buen ejemplo de la utilidad de este enfoque es el trabajo realizado en torno al mamut (Nogués-Bravo et al., 2008) donde se han podido evaluar las distintas hipótesis existentes para la desaparición de la especie y definir la más fuerte según la evidencia que aporta la modelación de su paleodistribucion en relación a la variación del espacio climático.

\section{Biogeografía de la conservación, usando los modelos como herramientas para la planificación}

Se ha identificado a los modelos de distribución de especies/ecosistemas como un requerimiento clave para la biogeografía de la conservación (Richardson \& Whitakker, 2010), el mayor uso que se les ha dado a los modelos de distribución es como sustitutos de la biodiversidad de un lugar determinado. Esto se ha realizado mediante dos perspectivas, la primera es sumando los modelos individuales, generando ensambles de modelos 
para determinar de forma indirecta la riqueza de especies de un lugar, y la segunda busca identificar la distribución de especies con algún problema de conservación o con alguna característica particular, definiendo así áreas de mayor valor donde se deberían concentrar los esfuerzos de protección. El objetivo de ambos enfoques es el mismo: utilizar los modelos para la identificación espacial de las zonas de mayor valor de protección y evaluar los vacíos espaciales de los esfuerzos de protección y conservación existentes; este enfoque se denomina también "análisis de vacíos" (Urbina-Cardona \& Flores-Villela, 2010).

El desarrollo de modelos más dinámicos que dan cuenta de los movimientos de las especies o del cambio climático, le está dando un nuevo valor al rol de los modelos de distribución dentro de los procesos de planificación de conservación (Loiselle et al., 2003; Wilson et al., 2005; Elith \& Leathwick, 2009; Richardson et al., 2010). Así como es posible modelar la distribución en diferentes tiempos y con diferentes restricciones espaciales (dispersión, migración), se puede también evaluar de qué forma las redes de protección existentes van cumpliendo las metas de conservación, generando diseño de redes de protección dinámicos que se adapten tanto en el tiempo como en el espacio a la variación de los rangos de distribución (Vos et al., 2008; Coetzee et al., 2009). En esta línea los programas para generar modelos están tendiendo a complementarse y fusionarse con los de priorización de áreas como ZONATION (Moilanen et al., 2008), lo que posibilita avizorar nuevos desarrollos y herramientas que permitirán definir nuevas áreas de protección y evaluar las ya existentes de forma más sistemática.

\section{Ejemplo de modelo de distribución, distribución actual y futura del belloto del norte}

Para ejemplificar cómo funcionan los modelos de distribución, a continuación se presenta la caracterización de la distribución potencial actual del belloto del norte (Beilschmiedia miersii) y su proyección bajo un escenario de cambio climático para el año 2050. Para generar el modelo de distribución del belloto del norte se definió como área de estudio el territorio chileno, ubicado entre los $30^{\circ}$ y los $34^{\circ}$ latitud Sur. Se utilizó como información de presencias los datos recopilados en el proyecto "Estudio sobre distribución geográfica y estado de conservación del belloto del norte (Beilschmiedia miersii)" (CICA Ingenieros Consultores, 2002). Como variables descriptoras se usaron las 19 variables bioclimáticas del proyecto Worldclim (Hijmans et al., 2005). Estas superficies climáticas cuentan con una resolución espacial de $1 \mathrm{~km}$ y corresponden a índices bioclimáticos que tienen relevancia directa en la ecología de las especies vegetales (Busby, 1991).

Para la modelación se usó BIOMOD 1.1-5 (Thuiller et al., 2009), utilizando 7 técnicas disponibles en este software (ANN, CTA, GAM, GBM, GLM, MARS, RF $\left.{ }^{6}\right)$. Se generaron 10.000 pseudoausencias distribuidas al azar dentro del área de estudio. Se estableció un porcentaje del 70\%-30\% para definir los sets de calibración y evaluación. El modelo binario final se determinó usando como valor de corte el umbral balanceado del ensamble final (Liu et al., 2005). El modelo futuro se obtuvo siguiendo los mismos pasos en BIOMOD, pero se actualizaron las variables descriptoras según el modelo de cambio climático HADCM3 (Gordon et al., 2000) bajo el escenario IPCC de emisión A2 (IPCC, 2007). Como primer paso se analizó la correlación espacial de las 19 variables bioclimáticas en el área de estudio. Para esto se calculó la correlación spearman entre pares de variables, descartando aquellas que tuviesen valores de correlación sobre 0,8 . Se obtuvo un total de seis variables con menor correlación entre sí: rango diurno promedio, isotermalidad, temperatura mínima del mes más frío, rango de temperatura anual, temperatura promedio del cuarto más cálido, precipitación anual. Para la evaluación del modelo se calcularon los valores de AUC, Kappa y TSS de cada técnica y se utilizó Random Forest (RF) para la determinación de las

\footnotetext{
6 Ver Cuadro $N^{0} 1$ para nombres, referencias y evaluación de los métodos.
} 
variables con mayor importancia en el modelo final (Breiman, 2001). Los valores de evaluación AUC para todas las técnicas fueron superiores a 0,75, siendo la técnica GLM la más baja y la con mayor valor RF $(0,84)$. Las variables de mayor relevancia para el modelo final fueron la temperatura promedio del cuarto más cálido y la precipitación anual. En la Figura $N^{\circ} 2$ se comparan los valores de ambas variables, donde se puede interpretar el aumento de las temperaturas promedias del cuarto más cálido y la disminución de la precipitación anual, según el escenario de cambio climático determinado.

La Figura $N^{\circ} 3$ muestra el resultado final del modelo binario y la proyección bajo el escenario de cambio climático HADCM3 A2. Como se observa, los resultados muestran una reducción de la distribución actual, de una superficie de distribución potencial de
820.100 hectáreas, se disminuye en el futuro hasta una superficie de 200.700 hectáreas. Se contrae el rango hacia el centro de su distribución actual en la zona del valle del Aconcagua y hacia las zonas de mayor altitud en los Altos de Cantillana. En la Figura $\mathrm{N}^{\circ} 4$ se presenta un gráfico con la variación de la altitud entre el modelo actual y futuro, indicada anteriormente. Es importante señalar que en el caso de este ejemplo se utilizaron solo variables climáticas para la definición del espacio ambiental del belloto del norte, si se incluyen en este modelo otro tipo de restricciones, como por ejemplo mecanismos de dispersión de la especie, el modelo podría ser aún más restringido que lo obtenido. Por lo que se debe señalar que este modelo se refiere a la proyección del espacio climático de la especie, por lo tanto, los análisis y evaluaciones tienen que estar en relación a esta proyección.

Figura $\mathrm{N}^{\circ} 2$

Comparación de las variables más importantes para el modelo de distribución potencial del belloto del norte*

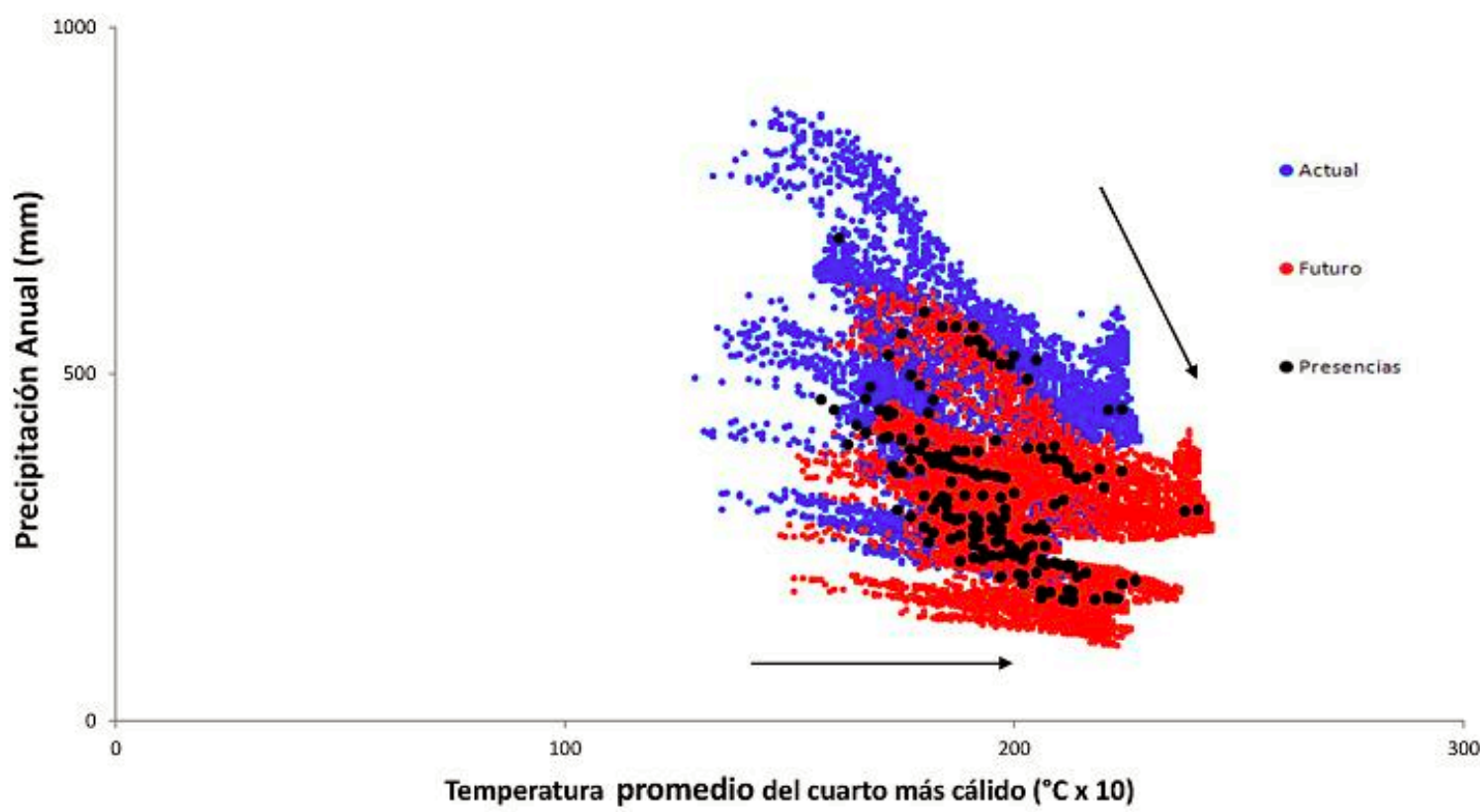

* Las flechas indican la dirección de cambio entre los valores actuales (oscuros) y los valores futuros (claros). Fuente: Elaboración propia. 
Figura $\mathrm{N}^{\circ} 3$

Modelos de distribución potencial actual (izquierda) y modelos de distribución proyectado bajo escenario de cambio climático para el año 2080 (derecha), para el belloto del norte

(Beilschmiedia miersii)
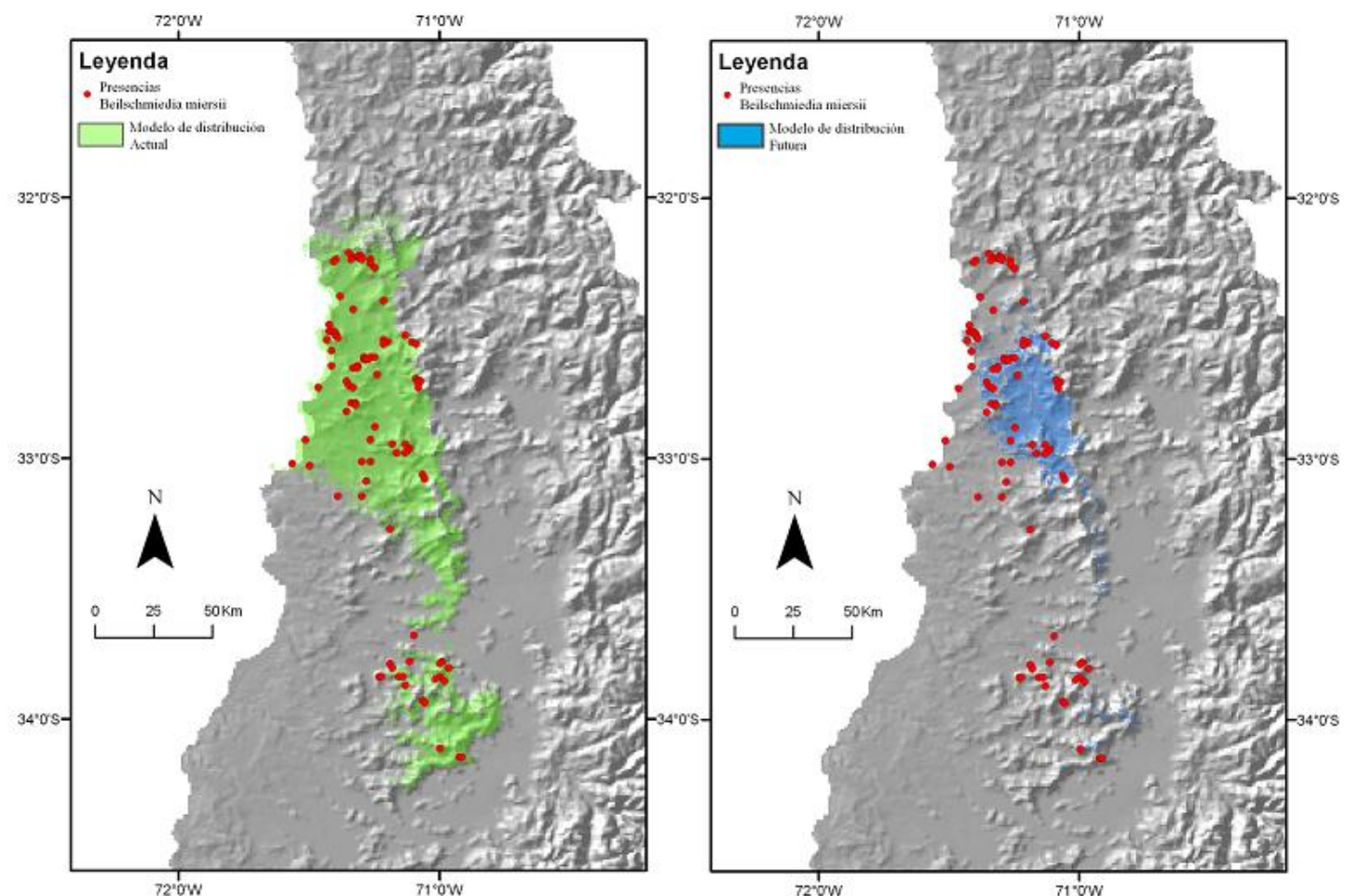

Fuente: Elaboración propia.

Figura $\mathrm{N}^{\circ} 4$

Variación de la altitud de la distribución potencial actual y proyectada según los modelos de distribución, para el belloto del norte (Beilschmiedia miersii)

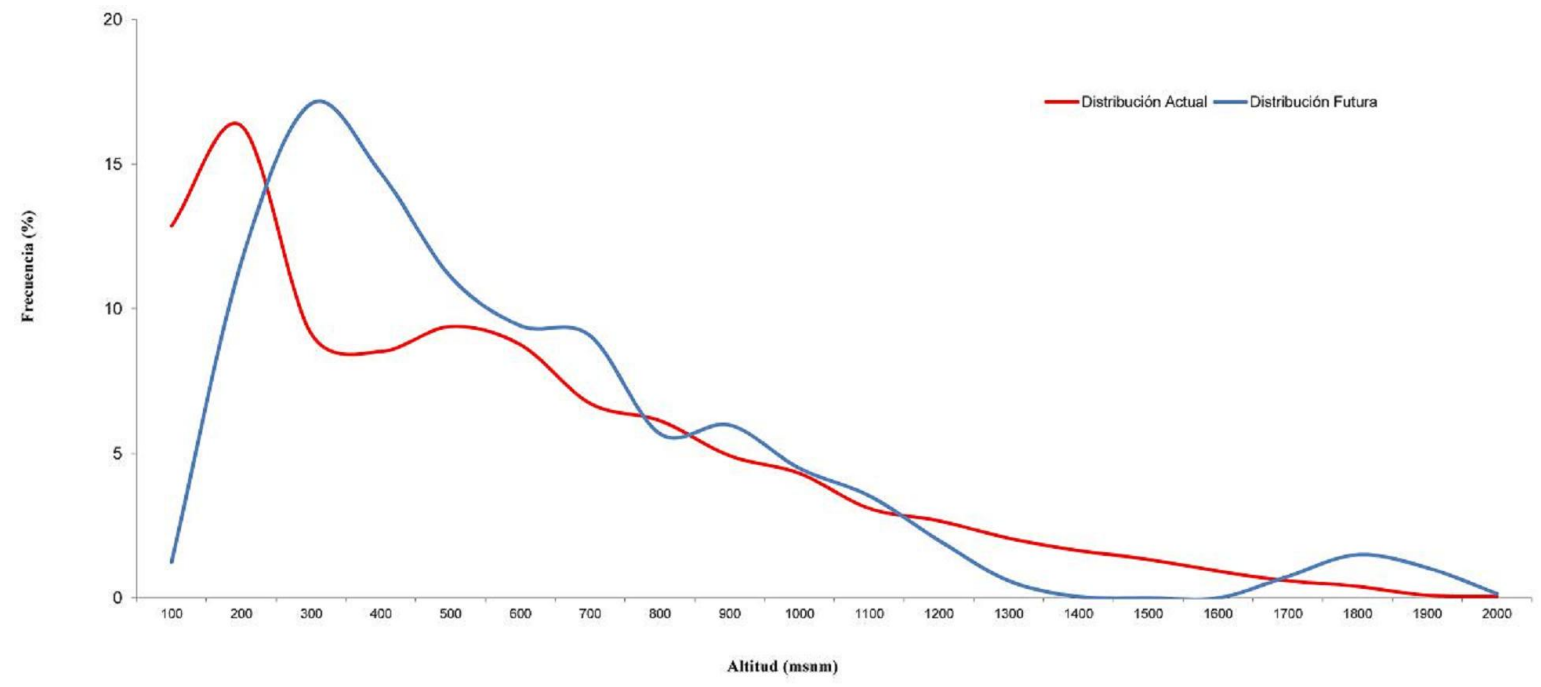

Fuente: Elaboración propia. 


\section{Consideraciones finales}

Se ha presentado una revisión general de los métodos, técnicas y aplicaciones asociadas a la generación de modelos de distribución desde un enfoque tanto a nivel de especies como de ecosistemas. De la revisión realizada se comprueba que es un campo en creciente desarrollo y que se ha involucrado en un amplio espectro de disciplinas, aportando nuevas herramientas para abordar preguntas asociadas a la distribución espacial y temporal de las especies y de los ecosistemas. La biogeografía se ha visto beneficiada por estas técnicas, desarrollando el enfoque predictivo de la disciplina tanto en términos de proyecciones futuras como en la mirada hacia el pasado. Del ejemplo presentado, se comprueba la utilidad de estos métodos para representar espacialmente la distribución actual de las especies. Dando cuenta, para el caso de esta especie arbórea endémica a la zona central de Chile, del patrón de reducción del rango de distribución actual hacia zonas de mayor altitud. Finalmente, los desafíos futuros que indican las nuevas direcciones en el campo de los modelos de distribución (Zimmermann et al., 2010), dan cuenta de la incorporación de elementos dinámicos que permiten caracterizar de mejor forma el espacio ambiental, como es el caso de las interacciones biológicas y la incorporación de variables antrópicas como es el uso del suelo. La identificación de los patrones espaciales y temporales de la distribución de los seres vivos, el objetivo central de la biogeografía, retoma con estas técnicas una relevancia dentro de las ciencias biológicas y ambientales como una herramienta útil para analizar y evaluar los cambios globales que se desarrollan en el planeta.

\section{Referencias bibliográficas}

ALLOUCHE, O.; TSOAR, A. \& KADMON, $R$. Assessing the accuracy of species distribution models: prevalence, kappa and the true skill statistic (TSS). Journal of Applied Ecology, 2006, vol. 43, p.1223-1232.

ARAUJO, M. B. \& NEW, M. Ensemble forecasting of species distributions. Trends in Ecology and Evolution, 2007, vol. 22, p. 42-47.
ASHCROFT, M. B. Identifying refugia from climate change. Journal of Biogeography, 2010, vol. 37, p. 1407-1413.

AUSTIN, M. P. Models for the analysis of species' response to environmental gradients. Vegetatio, 1987, vol. 69, p. 35-45.

AUSTIN, M. P. Species distribution models and ecological theory: a critical assessment and some possible new approaches. Ecological Modelling, 2007, vol. 200, p. 1-19.

AUSTIN, M. P.; NICHOLLS, A. O. \& MARGULES, C. R. Measurement of the realized qualitative niche: environmental niches of five Eucalyptus species. Ecological Monograph, 1990, vol. 60, p. 161-177.

AXELIUS, B. Areas of distribution and areas of endemism. Cladistics, 1991, vol. 7, p. 197-199.

BALDWIN, R. A. Use of maximum entropy modeling in wildlife research. Entropy, 2009, vol. 11, p. 854-866.

BARBET-MASSIN, M.; WALTHER, B. A.; THUILLER, W.; RAHBEK, C. \& JIGUET, F. Potential impacts of climate change on the winter distribution of Afro-Palearctic migrant passerines. Biology Letters, 2009, vol. 23, № 5(2), p. 248-251.

BEAUMONT, L.; HUGHES, L. \& POULSEN, M. Predicting species distributions: use of climatic parameters in BIOCLIM and its impact on predictions of species' current and future distributions. Ecological Modelling, 2005, vol. 10, p. 250268.

BENITO GARZÓN, M.; SÁNCHEZ DE DIOS, R. \& SAINZ OLLERO, H. Predictive modelling of tree species distributions on the Iberian Peninsula during the Last Glacial Maximum and Mid-Holocene. Ecography, 2007, vol. 30, N 1, p. 120-134.

BOX, E. O. Macroclimate and plant forms: an introduction to predictive modeling in phytogeography. The Hague: Junk, 1981.

BREIMAN, L. Random forest. Machine Learning, 2001, vol. 45, № 1, p. 5-32. 
BUSBY, J. R. BIOCLIM A bioclimate analysis and prediction system. In: MARGULES, C. R. \& AUSTIN, M. P. (eds.). Nature conservation: cost effective biological surveys and data analysis. Canberra: CSIRO, 1991, p. 64-68.

CARNAVAL, A. C.; HICKERSON, M. J.; HADDAD, C. F. B.; RODRIGUES, M. T. \& MORITZ, C. Stability predicts genetic diversity in the brazilian atlantic forest hotspot science. Science, 2009, vol. 323, Nº 5915, p. 785-789.

CICA INGENIEROS CONSULTORES. Estudio distribución geográfica y estado de conservación del Belloto del Norte (Beilschmiedia miersii). Santiago: CICA, Informe final, 2002.

COETZEE, B. W. T.; ROBERTSON, M. P.; ERASMUS, B. F. N.; VAN RENSBURG, B. J. \& THUILLER, W. Ensemble models predict important bird areas in southern Africa will become less effective for conserving endemic birds under climate change. Global Ecology and Biogeography, 2009, vol. 18, p. 701710 .

DEBLAUWE, V.; BARBIER, N.; COUTERON, P.; LEJEUNE, O.; \& BOGAERT, J. The global biogeography of semi-arid periodic vegetation patterns. Global Ecology and Biogeography, 2008, vol. 17, p. 715-723.

DODD, L. E. \& PEPE, M. S. Partial AUC estimation and regression. Biometrics, 2003, vol. 59, p. 614-623.

DORMANN, C.F. Promising the future? Global change projections of species distributions. Basic and Applied Ecology, 2007, vol. 8, p. 387-397.

DORMANN, C.; PURSCHKE, O.; GARCÍA MÁRQUEZ, J. R.; LAUTENBACH， S. \& SCHRÖDER, B. Components of uncertainty in species distribution analysis: a case study of the great grey shrike. Ecology, 2008, vol. 89, p. 3371-3386.

EDWARDS, T. C.; CUTLES, D. R.; ZIMMERMANN, N. E.; GEISER，L. \& MOISEN, G. G. Effects of sample survey Design on the accuracy of classification three models in species distribution models. Ecological Modelling, 2006, vol. 199, p. 132-141.

ELITH, J.; BURGMAN, A. \& REGAN, $H$. Mapping epistemic uncertainties and vague concepts in predictions of species distribution. Ecological Modelling, 2002, vol. 157, p. 313-329.

ELITH， J.; GRAHAM, C. H. ; ANDERSON, R. P.; DUDÍK, M.; FERRIER, S.; GUISAN, A.; HIJMANS, R. J.; HUETTMANN, F.; LEATHWICK, J. R.; LEHMANN, A.; LI, J.; LOHMANN, L. G.; LOISELLE, B. A.; MANION, G.; MORITZ, C.; NAKAMURA, M.; NAKAZAWA, Y.; OVERTON, J. M.; PETERSON, A. T.; PHILLIPS, S. J.; RICHARDSON, K. S.; SCACHETTI-PEREIRA, R.; SCHAPIRE, R. E.; SOBERÓN, J.; WILLIAMS, S.; WISZ, M. S. \& ZIMMERMANN, N. E. Novel methods improve prediction of species' distributions from occurrence data. Ecography, 2006, vol. 29, p. 129-151.

ELITH, J. \& GRAHAM, C. H. Do they? How do they? Why they differ? On finding reasons for differing performance of species distribution models. Ecography, 2009, vol. 32 , p. 66-77.

ELITH, J. \& LEATHWICK, J. Species distribution models: ecological explanation and predictionacross space and time. Annual Review of Ecology, Evolution and Systematic, 2009, vol. 40, p. 677-697.

ELITH, J.; KEARNEY, M. \& PHILLIPS, S. The art of modelling range-shifting species. Methods in Ecology and Evolution, 2010, vol. 1, p. 330-342.

ELITH, J.; PHILLIPS, S.; HASTIE, T.; DUDIK, M.; CHEE, Y. \& YATES, C. A statistical explanation of MaxEnt for ecologists. Diversity and Distributions, 2011, vol. 17, p. 43-57.

EVANS, M. E. K.; SMITH, S. A.; FLYNN, R. \& DONOGHUE, M. J. Climate, niche evolution, and diversification of the "birdcage" evening primroses (Oenothera, sections Anogra and Kleinia). American Naturalist, 2009, vol. 173, p. 225-240. 
FEELEY, K. J. \& SILMAN, M. R. Modelling Andean and Amazonian plant species responses to climate change: the effects of geo-referencing errors and the importance of data filtering. Journal of Biogeography, 2009, vol. 37 , p. 733-740.

FERRIER, S.; MANION, G.; ELITH, J. \& RICHARDSON, K. Using generalized dissimilarity modelling to analyse and predict patterns of beta diversity in regional biodiversity assessment. Diversity and Distributions, 2007, vol. 13, p. 252-264.

FICETOLA, G. F.; MAIORANO, L.; FALCUCCI, A.; DENDONCKER, N.; BOITANI, L.; PADOA-SCHIOPPA, E.; MIAUD, C. \& THUILLER, W. Knowing the past to predict the future: land-use change and the spread of invasive bullfrogs. Global Change Biology, 2010, vol. 16, p. 528-537.

FLANTUA, S. G. A.; VAN BOXEL, J. H.; HOOGHIERNSTRA, H. \& VAN SMAALEN, J. Application of GIS and logistic regression to fossil pollen data in modelling present and past spatial distribution of the Colombian savanna. Climate Dynamics, 2007, vol. 29, p. 697-712.

FRANKLIN, J. Mapping species distributions: spatial inference and prediction. Cambridge: Cambridge University Press, 2009.

FREEMAN, E. A. \& MOISEN, G. G. A comparison of the performance of threshold criteria for binary classification in terms of predicted prevalence and kappa. Ecological Modelling, 2008, vol. 217, p. 48-58.

FRIEDMAN, J. Multivariate adaptive regression splines (with discussion). Annals of Statistics, 1991, vol. 19, p. 1-141.

FRIEDMAN, J. Greedy function approximation: a gradient boosting machine. Annals of Statistics, 2001, vol. 29, p. 1189 1232.

GORDON, C.; COOPER, C.; SENIOR, C. A.; BANKS, H. T.; GREGORY, J. M.; JOHNS, T. C.; MITCHELL, J. F. B. \& WOOD, R. A. The simulation of SST, sea ice extents and ocean heat transports in a version of the
Hadley Centre coupled model without flux adjustments. Climate Dynamics, 2000, vol. 16, p. 147-168.

GRAHAM, C. H.; VANDERWAL, J.; PHILLIPS, S. J.; MORITZ, C. \& WILLIAMS, S. E. Dynamic refugia and species persistence: tracking spatial shifts in habitat through time. Ecography, 2010, vol. 33, p. 10621069.

GUISAN, A. \& ZIMMERMANN, N. E. Predictive habitat distribution models in ecology. Ecoogical Modeling, 2000, vol. 135, p. 147-186.

GUISAN, A.; EDWARDS, J. R. \& HASTIE, T. Generalized linear and generalized additive models in studies of species distribution: Setting the scene. Ecological Modelling, 2002, vol. 157, p. 89-100.

GUISAN, A. \& THUILLER, W. Predicting species distribution: offering more than simple habitat models. Ecology Letters, 2005, vol. 8, p. 993-1009.

HASTIE, T. J. \& TIBSHIRANI, R. Generalized additive models. London: Chapman \& Hall, 1990.

HERNÁNDEZ, P. A.; GRAHAM, C. H.; MASTER, L. L. \& ALBERT, D. L. The effect of sample size and species characteristics on performance of different species distribution modeling methods. Ecography, 2006, vol. 29, p. 773-785.

HIJMANS, R. J.; CAMERON, S. E.; PARRA, J. L.; JONES, P. G. \& JARVIS, A. Very high resolution interpolated climate surfaces for global land areas. International Journal of Climatology, 2005, vol. 25, p. 1965-1978.

HIJMANS, R. J. \& GRAHAM, C. H. The ability of climate envelope models to predict the effect of climate change on species distributions. Global Change Biology, 2006, vol. 12, p. 2272-2281.

HIRZEL, A. H.; HAUSSER, J.; CHESSEL, D. \& PERRIN, N. Ecological-niche factor analysis: How to compute habitat- suitability maps without absence data? Ecology, 2002, vol. 83, p. 2027-2036. 
HIRZEL, A. H.; LE LAY, G.; HELFER, V.; RANDIN, C. \& GUISAN, A. Evaluating the ability of habitat suitability models to predict species presences. Ecological Modelling, 2006, vol. 199, N², p. 142-152.

HUTCHINSON, G. E. Concluding remarks. Cold Spring Harbour Symposium on Quantitative Biology, 1957, vol. 22, p. 415-427.

IPCC. Climate Change 2007: impacts, adaptation, and vulnerability. In: PARRY, M. L.; CANZIANI, O. F.; PALUTIKOF, J. P.; VAN DER LINDEN, P. J. \& HANSON, C. E. (eds.). Contribution of working group II to the Fourth Assessment Report of the Intergovernmental Panel on Climate Change. Cambridge: Cambridge University Press, 2007, p. Xx-xx.

JIMENEZ-VALVERDE, A.\& LOBO, J. M. Threshold criteria for conversion of probability of species presence to either- or presence-absence. Acta Oecologica, 2007, vol. 31, p. 361-369.

JIMENEZ-VALVERDE, A.; LOBO, J. M. \& HORTAL, J. Not as good as they seem: the importance of concepts in species distribution modelling. Diversity and Distributions, 2008, vol. 14 , p. 885-890.

KADMON, R.; FARBER, O. \& DANIN, A. A systematic analysis of factors affecting the performance of climatic envelope models. Ecological Applications, 2003, vol. 13, p. 853-867.

KESSELL, S. R. Gradient modeling: resource and fire management. New York: Springer Verlag, 1979.

KOZAK, K. H.; GRAHAM, C. H. \& WIENS, J. J. Integrating GIS-based environmental data into evolutionary biology. Trends in Ecology and Evolution, 2008, vol. 23, p. 141-148.

LE LAY, G.; ENGLER, R.; FRANC, E. \& GUISAN, A. Prospective sampling based on model ensembles improves the detection of rare species. Ecography, 2010, vol. 33, p. 1015-1027.

LE MAÎTRE, D. C.; THUILLER, W. \& SCHONEGEVEL, L. Developing an approach to defining the potential distributions of invasive plant species: a case study of Hakea species in South Africa. Global Ecology and Biogeography, 2008, vol. 17, p. 569-584.

LEK, S.; DELACOSTE, M.; BARAN, P.; DIMOPOULOS, I.; LAUGA, J. \& AULAGNER, $S$. Application of neural networks to modelling nonlinear relationships in ecology. Ecological Modelling, 1996, vol. 90, p. 39-52.

LIU, C.; BERRY, P. M.; DAWSON, T. P. \& PEARSON, R. G. Selecting thresholds of occurrence in the prediction of species distributions. Ecography, 2005, vol. 28, p. 385-393.

LOBO, J. M.; JIMÉNEZ-VALVERDE, A. \& REAL, R. AUC: a misleading measure of the performance of predictive distribution models. Global Ecology and Biogeography, 2008, vol. 17, No2, p. 145-151.

LOISELLE, B. A.; HOWELL, C. A.; GRAHAM, C. H.; GOERCK, J. M.; BROOKS, T.; SMITH, K. G. \& WILLIAMS, P. H. Avoiding pitfalls of using species distribution models in conservation planning. Conservation Biology, 2003, vol. 17, p. 1591-1600.

LOMOLINO, M. V.; RIDDLE, B. R.; WHITTAKER, R. J. \& BROWN, J. H. Biogeography. Sunderland: Sinauer, 2010.

MACARTHUR, R. H. Geographical Ecology. New York: Harper and Row, 1972.

MANEL, S.; WILLIAMS, H. C. \& ORMEROD, S. J. Evaluating presenceabsence models in ecology: the need to account for prevalence. Journal of Applied Ecology, 2001, vol. 38, p. 921-931.

MARINI, M. A.; BARBET-MASSIN, M.; MARTÍNEZ, J.; PRESTES, N. P. \& JIGUET F. Applying ecological niche modelling to plan conservation actions for the Red-spectacled Amazon (Amazona pretrei). Biological Conservation, 2010, vol. 143, p. 102-112.

MARMION, M.; PARVIAINEN, M.; LUOTO, M.; HEIKKINEN, R. K. \& THUILLER, W. Evaluation of consensus methods in predictive species distribution modelling. Diversity and Distributions, 2009, vol. 15, p. 59-69. 
MARTÍNEZ-MEYER, E.; PETERSON, A. T. \& HARGROVE, W. Ecological niches as stable distributional constraints on mammal species, with implications for Pleistocene extinctions and climate projections for biodiversity. Global Ecology and Biogeography, 2004, vol. 13, p. 305-314.

MCCULLAGH, P. \& NELDER, J. A. Generalized linear models. London: Chapman and Hall, 1989.

MCGILL, B. Matters of scale. Science, 2010, vol. 328, p. 575-576.

MEYNARD, C. N. \& QUINN, J. F. Predicting species distributions: a critical comparison of the most common statistical models using artificial species. Journal of Biogeography, 2007, vol. 34, p. 1455-1469.

MOILANEN, A.; LEATHWICK, J. R. \& ELITH, J. A method for freshwater conservation prioritization. Freshwater Biology, 2008, vol. 53, p. 577-592.

MORRONE, J. J. Evolutionary biogeography: an integrative approach with case studies. New York: Columbia University Press, 2009.

NEWBOLD, T. Applications and limitations of museum data for conservation and ecology, with particular attention to species distribution models. Progress in Physical Geography, 2010, vol. 34, p. 3-22.

NIX, H. A biogeographic analysis of Australian elapid snakes. In: LONGMORE, R. (ed.). Atlas of elapid snakes of Australia. Canberra: Australian Government Publishing Service, 1986, p. 4-5.

NOGUÉS-BRAVO, D.; RODRÍGUEZ, J.; HORTAL, J.; BATRA, P. \& ARAÚJO, M. B. Climate change, humans, and the extinction of the woolly mammoth. PLoS Biology, 2008, vol. $6, N^{\circ} 4$, p. 685-692.

NOGUÉS-BRAVO, D. Predicting the past distribution of species' climatic niches. Global Ecology and Biogeography, 2009, vol. 18, p. 521-531.

PARVIAINEN, M.; LUOTO, M. \& HEIKKINEN, R. K. The role of local and landscape level measures of greenness in modelling boreal plant species richness. Ecological Modeling, 2009, vol. 220, p. 26902701.

PEARMAN, P.; RANDIN, C.; BROENNIMANN, O.; VITTOZ, P.; VAN DER KNAAP, W.; ENGLER, R.; LE LAY, G.; ZIMMERMANN, N. E. \& GUISAN, A. Prediction of plant species distributions across six millennia. Ecology Letters, 2008a, vol. 11, p. 357-369.

PEARMAN, P.; GUISAN, A.; BROENNIMANN, O. \& RANDIN, C. Niche dynamics in space and time. Trends in Ecology and Evolution, 2008b, vol. 23, №3, p. 149-158.

PETERSON, A. T.; PAPE, M. \& EATON, $M$. Transferability and model evaluation in ecological niche modeling: A comparison of GARP and Maxent. Ecography, 2007, vol. 30, p. 550-560.

PETERSON, A. T. \& NYARI, Á. Ecological niche conservatism and Pleistocene refugia in the thrush-like mourner, Schiffornis sp., in the Neotropics. Evolution, 2008, vol. 62, p. 173-183.

PHILLIPS, S. J.; ANDERSON, R. P. \& SCHAPIRE, R. E. Maximum entropy modelling of species geographic distributions. Ecological Modeling, 2006, vol. 190, p. 231-259.

PRASAD, A. M.; IVERSON, L. R. \& LIAW, A. Newer classification and regression tree techniques: Bagging and random forests for ecological prediction. Ecosystems, 2006, vol. 9, p. 181-199.

RANDIN, C. F.; JACCARD, H.; VITTOZ, P.; YOCCOZ, N. G. \& GUISAN, A. Land use improves spatial predictions of mountain plant abundance but not presence-absence. Journal of Vegetation Science, 2009a, vol. 20, $\mathrm{N}^{\circ}$ 6, p. 996-1008.

RANDIN, C. F.; ENGLER, R.; NORMAND, S.; ZAPPA, M.; ZIMMERMANN, N. E.; PEARMAN, P. B.; VITTOZ, P.; THUILLER, W. \& GUISAN, A. Climate change and plant distribution: local models predict highelevation persistence. Global Change Biology, 2009b, vol. 15, Nº, p. 1557-1569. 
RICHARDSON, D. M. \& WHITTAKER, R. J. Conservation biogeography-foundations, concepts and challenges. Diversity and Distributions, 2010, vol. 16, №3, p. 313-320.

RICHARDSON, D. M.; IPONGA, D. M.; ROURA-PASCUAL, N.; KRUG, R. N.; MILTON, S. J.; HUGHES, G. O. \& THUILLER,W. Accommodating scenarios of climate change and management in modelling the distribution of the invasive tree Schinus molle in South Africa. Ecography, 2010, vol. 33, p. 1049-1061.

RIORDAN, E. C. \& RUNDEL, P. W. Modelling the distribution of a threatened habitat: the California sage scrub. Journal of Biogeography, 2009, vol. 36, p. 2176-2188.

ROSE, N. A. \& BURTON, P. J. Using Bioclimatic Envelopes to Identify Temporal Corridors in Support of Conservation Planning in a Changing Climate. Forest Ecology and Management, 2010, vol. 258, p. S64-S74.

SEGURADO, P. \& ARAUJO, M. B. An evaluation of methods for modeling species distributions. Journal of Biogeography, 2004, vol. 31 , p. $1555-1568$.

SINCLAIR, S. J.; WHITE, M. D. \& NEWELL. G. R. How useful are species distribution models for managing biodiversity under future climates? Ecology and Society, 2010, vol. 15, N 1, p. 8.

SMITH, S. A. \& DONOGHUE, M. J. Combining Historical Biogeography with Niche Modeling in the Caprifolium Clade of Lonicera (Caprifoliaceae, Dipsacales). Systematic Biology, 2010, vol. 590, p. 322341.

STOCKWELL, D. \& PETERS, D. The GARP modelling system: problems and solutions to automated spatial prediction. International Journal of Geographical Information Science, 1999, vol. 13, p. 143-158.

SWENSON, N. G. The past and future influence of geographic information systems on hybrid zone, phylogeographic and speciation research. Journal of Evolutionary Biology, 2008, vol. 21, p. 421-434.
THUILLER, W.; RICHARDSON, D. M.; PYSEK, P.; MIDGLEY, G. F.; HUGHES, G. O. \& ROUGET, M. Niche-based modelling as a tool for predicting the risk of alien plant invasions at a global scale. Global Change Biology, 2005, vol. 11, p. 2234-2250.

THUILLER, W.; LAFOURCADE, B.; ENGLER R. \& ARAUJO, M. BIOMOD a platform for ensamble forecasting of species distributions. Ecography, 2009, vol. 32, p. 369-373.

TSOAR, A.; ALLOUCHE, O.; STEINITZ, O.; ROTEM, D. \& KADMON, R. A comparative evaluation of presence-only methods for modelling species distribution. Diversity and Distributions, 2007, vol. 13, p. 397-405.

URBINA-CARDONA, J. N. \& FLORESVILLELA, O. Ecological-Niche Modeling and Prioritization of Conservation-Area Networks for Mexican Herpetofauna. Conservation Biology, 2010, vol. 24, N 1, p. 1031-1041.

VAN STRIEN, M. Best practice species distribution modelling protocol, ver.1.0. Switzerland: Spatial Ecology Group, University of Lausanne, 2008.

VAN NEIL, K. P.; LAFFAN, S. W. \& LEES, B. G. Effect of error in the DEM on environmental variables for predictive vegetation modelling. Journal of Vegetation Science, 2004, vol. 15, p. 747-756.

VAYSSIERES, M. P.; PLANT, R. E. \& ALLEN-DÍAZ, B. H. Classification trees: an alternative non-parametric approach for predicting species distributions. Journal of Vegetation Science, 2000, vol. 11, p. 679694.

VOS, C.; BERRY, P.; OPDAM, P.; BAVECO, H.; NIJHOF, B.; O'HANLEY, J.; BELL, C. \& KUIPERS, H. Adapting landscapes to climate change: examples of climate-proof ecosystem networks and priority adaptation zones. Journal of Applied Ecology, 2008, vol. 45, p. 1722-1731.

WIENS, J. J. \& GRAHAM, C. H. Niche conservatism: integrating evolution, ecology, and conservation biology. Annual Review of Ecology Evolution and Systematics, 2005, vol. 36, p. 519-539. 
WILLIAMS, J. W. \& JACKSON, S. T. Novel climates, no-analog plant communities, and ecological surprises: past and future. Frontiers in Ecology and Evolution, 2007, vol. 5, p. 475-482.

WILLIAMS, J. W.; JACKSON, S. T. \& KUTZBACH, J. E. Projected distributions of novel and disappearing climates by 2100AD. Proceedings of the National Academy of Sciences, 2007, vol. 104, p. 5738-5742.

WILLIS, S. G.; THOMAS, C. D.; HILL, J. K.; COLLINGHAM, Y. C.; TELFER, M. G.; FOX, R. \& HUNTLEY, B. Dynamic distribution modelling: predicting the present from the past. Ecography, 2009, vol. 32, p. 5-12.

WILSON, K. A.; WESTPHAL, M. I.; POSSINGHAM, H. P. \& ELITH, J. Sensitivity of conservation planning to different approaches to using predicted species distribution data. Biological Conservation, 2005, vol. 122, p. 99-112.

WHITTAKER, R. J.; ARAÚJO, M. B.; JEPSON, P.; LADLE, R. J.; WATSON, J. E. M. \& WILLIS, K. J. Conservation biogeography: assessment and prospect. Diversity and Distributions, 2005, vol. 11, p. 3-23.
WISZ, M. S.; HIJMANS, R. J. ; LI, J.; PETERSON, A. T.; GRAHAM, C. H. \& GUISAN, A. NCEAS Predicting Species Distributions Working Group. Effects of sample size on the performance of species distribution models. Diversity and Distributions, 2008, vol. 14, p. 763-773.

YESSON, C. \& CULHAM, A. Phyloclimatic modeling: combining phylogenetics and bioclimatic modeling. Systematic Biology, 2006, vol. 55, p. 785802.

ZIMMERMANN, N. E.; EDWARDS, T. C.; GRAHAM, C. H.; PEARMAN, P. \& SVENNING, J. C. New trends in species distribution modeling. Ecography, 2010, vol. 33, p. 985-989.

ZIMMERMANN, N. E.; EDWARDS, T. C.; MOISEN, G. G.; FRESCINO, T. S. \& BLACKARD, J. A. Remote sensing-based predictors improve distribution models of rare, early successional and broadleaf tree species in Utah. Journal of Applied Ecology, 2007, vol. 44, N 5, p. 1057-1067. 